\title{
Trivium
}

Revue franco-allemande de sciences humaines et sociales - Deutsch-französische Zeitschrift für Geistesund Sozialwissenschaften

1 | 2008

"Iconic Turn » et réflexion sociétale

\section{Par-delà le langage ? Remarques sur la logique des images}

\section{Gottfried Boehm}

Traducteur : Denis Trierweiler

\section{CpenEdition}

Journals

Édition électronique

URL : http://journals.openedition.org/trivium/252

DOI : $10.4000 /$ trivium.252

ISSN : 1963-1820

Éditeur

Les éditions de la Maison des sciences de l'Homme

Référence électronique

Gottfried Boehm, "Par-delà le langage ? Remarques sur la logique des images », Trivium [En ligne], 1 । 2008, mis en ligne le 08 avril 2008, consulté le 08 septembre 2020. URL : http://

journals.openedition.org/trivium/252 ; DOI : https://doi.org/10.4000/trivium.252

Ce document a été généré automatiquement le 8 septembre 2020.

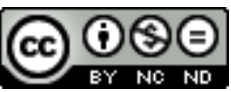

Les contenus des la revue Trivium sont mis à disposition selon les termes de la Licence Creative Commons Attribution - Pas d'Utilisation Commerciale - Pas de Modification 4.0 International. 


\title{
Par-delà le langage ? Remarques sur la logique des images
}

\author{
Gottfried Boehm
}

Traduction : Denis Trierweiler

\section{NOTE DE L'ÉDITEUR}

Pour des raisons de droits, il ne nous est pas possible d'utiliser les images de la première publication de l'article. Nous avons laissé la référence de l'emplacement des images manquantes en gras dans le corps du texte afin de les retrouver plus facilement dans le texte original.

Nous tenons par ailleurs à remercier chaleureusement Gottfried Boehm ainsi que les éditions DuMont (Cologne) de nous avoir accordé le droit de traduire son article.

\section{La question}

1 En dépit de son actualité, il n'est guère possible de faire avancer la question de l'image au moyen de diagnostics et de recettes par trop rapides. Lorsque tout le monde - ou presque - parle des "images", lorsque les disciplines les plus diverses les ont découvertes, il est alors nécessaire de s'entendre sur les prémisses. Si le « tournant vers l'image " que décrit le concept d'iconic turn existe réellement, alors ce ne sont pas seulement des phénomènes ponctuels ou superficiels qui sont en jeu mais des présupposés fondamentaux de notre culture. En ce sens, je voudrais ici discuter la thèse suivante: les images possèdent une logique propre qui n'appartient qu'à elles. Par logique, nous entendons l'engendrement cohérent de sens à partir d'authentiques moyens iconiques. Et pour explication, j'ajoute que cette logique est non prédicative, ce qui veut dire qu'elle n'est pas formée sur le modèle de la proposition ou d'autres formes langagières. Elle n'est pas parlée mais réalisée en étant perçue. 
2 Les conséquences de ce postulat sont hautement sujettes à controverse et nous conduisent sur un terrain difficile. On sait que les images possèdent une force et un sens propres. Ce savoir est vieux comme le monde et il a été partagé par bien des hommes depuis la Préhistoire (fig. 1).

3 Il est autre chose, bien sûr, que de comprendre comment cette production de sens iconique fonctionne. En dépit de 2500 années de science européenne, ce problème est demeuré étrangement marginalisé. Ce n'est que depuis peu que l'on s'est attelé au projet d'élaborer une "science de l'image ", alors que le langage peut se targuer d'une approche discursive constante depuis le Cratyle $^{1}$ de Platon. Mais entre-temps, la situation s'est dramatiquement modifiée: des images jusque-là inconnues sont apparues dans le laboratoire de la modernité. La révolution digitale a développé des moyens de communication iconiques globaux; des procédés imageants veillent à ce que les sciences de la nature puissent acquérir, avec des instruments d'imagerie, des savoirs qui ne peuvent être obtenus que par cette voie.

4 Même au-delà de l'art, les images sont irremplaçables, si bien que le discours sur l'image est devenu un véritable défi qui nécessite de l'instruction (enseignement). Il ne pourra pas y avoir de compétence iconique ni de critique de l'image, si le statut de l'iconique reste confus, et si les images restent sollicitées de toutes parts, sans que nous sachions pour autant avec suffisamment de précision comment elles fonctionnent (fig. 2).

5 Je ne cache pas que je considère cette question comme l'un des grands défis intellectuels du temps présent. Ainsi qu'il apparaîtra, il est lié à la critique du langage. Le but n'en est pas une nostalgie de l'absence de langage, ou l'analphabétisme d'une prétendue innocence visuelle des images, mais une nouvelle détermination relationnelle qui cesse de soumettre l'image au langage, qui élargit plutôt le logos pardelà sa verbalisation limitée, par la potentialité de l'iconique, et du même coup le transforme. Sur cette voie, nous parcourrons les étapes suivantes :

1. Que voulons-nous dire lorsque nous parlons de l'« image »?

2. Pourquoi la critique du langage sert-elle à la compréhension de l'image?

3. Que signifie : logique des images?

Les argumentations théoriques seront inévitables, mais elles se vérifieront constamment sur des images, parce que celles-ci, à la différence des médias, existent non pas en tant que technologies générales, mais dans une singularité caractéristique. Et c'est celle-ci que nous honorons le mieux en regardant des images.

\section{Traits fondamentaux de l'iconique}

6 Personne ne nous contredira sans doute, si nous accordons aux images un pouvoir sur le corps, l'âme et l'esprit. Mais comment prend-il naissance? Dans le sens d'une compréhension élémentaire, on pourra le ramener à un substrat matériel. Or, il est certain que les images ne se réduisent pas à la matière. Sur des surfaces, dans la poussière de la couleur, dans la pierre, sur du bois ou de la toile, sur des supports photosensibles ou sur des écrans digitaux, il y a toujours quelque chose d'autre qui se montre : une vue, un spectacle, un sens - une image en somme.

7 Son statut est tout aussi singulier qu'énigmatique : elle est à la fois chose et non-chose, elle se trouve à mi-chemin entre la pure tangibilité et des rêves éthérés : c'est le 
paradoxe d'une irréalité réelle. Et c'est là-dessus que prend appui la «sortie de l'image ", si souvent et si diversement pratiquée par les artistes au $\mathrm{xx}^{\mathrm{e}}$ siècle. Elle tente de dissoudre l'existence hybride de l'iconique en ses composantes: en un véritable «quelque chose », plus connu sous le nom de readymade, qui tente de se défaire de tout aspect représentatif, et en une pure image de l'imagination, quelque chose de cognitif, qui n'a qu'une faible assise matérielle. Ce qui donne à réfléchir, c'est combien il aura été difficile, ce faisant, de se débarrasser de l'imaginaire, de se libérer du fait que, d'une certaine manière, l'iconique était toujours présent. Même l'un des plus illustres représentants du Minimal Art américain, Donald Judd, qui voulait prendre les objets en tant qu'« objets ", inexpressifs, et qui y liait aussi - conformément à la maxime énoncée par Frank Stella: "What you see is what you see!»- un rite de purification de la perception, dut finalement reconnaître que même le fait le plus simple pouvait encore montrer quelque chose et que la force projective de la perception s'y enflammait également sur un mode iconique (fig. 3).

autres termes: l'image correspond manifestement à un besoin profondément ancré dans l'homme, et il suffit de quelques manipulations tout à fait élémentaires, pour que, dans le continuum discret du monde matériel, non seulement quelque chose " remonte ", mais pour qu'ici ou là quelque chose se "montre ", et que s'ouvre à l'œil une vue enracinée dans le matériel, un sens. Par conséquent, l'« iconique » repose sur une " différence » réalisée par le voir la vision. C'est elle qui fonde la possibilité de voir l'un à la lumière de l'autre, ou dans quelques traits, par exemple, une figure.

Déterminer quelque chose comme quelque chose est un acte créateur fondamental qui ne s'exerce pas seulement à un niveau linguistique, mais également entre l'œil et le monde matériel. Aussi faible que puisse sembler le seuil entre artefact visuel et simple objet dans le prétendu barbouillage de petits enfants, dans des créations archaïques ou dans le contexte de la modernité, au fond, il marque une césure sans pareille. Car, par la manifestation matérielle d'un immatériel, qui devient par là visible, se définit l'humanité. Pour autant que les découvertes préhistoriques l'attestent, son premier protagoniste fut un homo pictor, longtemps avant qu'il ne se déterminât, dans l'Antiquité, comme zoon logon echon.

10 Ce qui devient visible dans la différence iconique, le contenu qu'elle convoque, désigne quelque chose d'absent. Que l'adorant antique ait été un être réel ou imaginé, dans la représentation, il était un autre. Nulle image ne peut se défaire de ce décalage irrévocable, et aucune ne crée de la présence sans l'incontournable ombre de l'absence. Il n'est qu'à Pygmalion qu'il fut accordé, par intervention divine, de créer un véritable être vivant à partir de la sculpture féminine faite de sa main, et de reconduire l'irréalité en réalité. Dans un design utopique enfin, l'image peut être conçue comme un clone génétique, comme un double vivant. L'image dont nous parlons n'est cependant pas le simulacrum dépourvu de différence, et pas non plus le camouflage qui se fait disparaître lui-même, mais la différence d'un imaginaire. Maurice Merleau-Ponty disait qu'elle prête sa "chair » à un absent et ainsi lui confère de la présence. Le pouvoir qui peut habiter les images réside manifestement dans leur capacité à donner accès à quelque chose qui est mort ou ailleurs : un souverain puissant, un contenu religieux, quelque chose d'invisible qui n'a ni corps ni visage, ou bien quelque chose d'imaginé, de rêvé. Le pouvoir de l'image signifie : « [elle] fait voir $»{ }^{2}$ elle ouvre les yeux, elle montre.

11 Sans doute Leonard de Vinci avait-il à l'esprit quelque chose de l'ordre de ce pouvoir des images lorsqu'il confronta ses élèves de l'école de peinture à l'étude de la « macchia 
", c'est-à-dire avec des taches arbitraires, telles que l'on peut les observer par exemple sur le crépi de vieux murs. Il invita ses élèves à voir dans ces formations - à l'instar des taches du test de Rorschach - à l'aide de leur imagination des paysages, des visages, des figures, des monstres etc. Une couleur de surface devient ainsi une chose à double-fond que le regard est capable de percevoir comme une image, grâce à ses possibilités constructives du « voir en tant que » et du « voir dans ». Léonard a cependant limité sa découverte de l'effet schématisant des taches à l'entraînement imaginatif de l'artiste, sans considérer déjà les taches elles-mêmes comme des images.

Une foule de paradigmes, de mythes, de légendes et de métaphores éclairent cette scène primitive de l'immatérialisation du matériel: Narcisse et autres héros du spéculaire, Pygmalion, la Méduse, Dédale-Icare, les légendes d'Abgar et de Véronique, mais aussi ces récits chinois qui rapportent comment le peintre lui-même entre dans l'image qu'il a produite pour y disparaître corps et âme. Ce sont tout particulièrement le reflet et l'empreinte - par exemple celle de la tête du Christ sur le linceul - qui s'approchent de formes d'images spontanées telles que la nature en produit aussi. Mais seul un œil intelligent est capable de lire les deux comme des images. Voici suffisamment d'exemples qui montrent que la praxis de l'image, la haute estime en laquelle sont tenues les images ainsi que la réflexion qui les accompagne, font partie des grandes conquêtes culturelles. Et cependant, dans le passé, le paradigme de l'image n'a guère été en mesure de s'octroyer un lieu légitime dans la hiérarchie du savoir.

\section{Critique du langage}

Les raisons pour lesquelles les discours iconiques furent marginalisés - même si ce ne fut pas le cas entre artistes et critiques d'art, mais bien dans le champ des sciences sont à chercher dans une certaine compréhension du langage. Celui qui cherche à saisir la logique des images, dans le sens de notre thèse, butera immanquablement sur l'ombre du langage qui s'étend par-dessus l'iconique. Celle-ci existe en divers degrés, mais avant de m'intéresser à la façon dont la question est généralement abordée aujourd'hui, je vais au préalable désigner trois paradigmes lourds de conséquences.

1. Sans doute le plus ancien document de théorie de l'image de notre culture est le décalogue formulé dans le livre de l'Exode de l'Ancien Testament. L'adepte s'y voit imposé - démarche tout à fait étonnante - une praxis négative de l'image : Tu ne feras pas d'image, de représentation ou d'allégorie du Très-Haut! En d'autres termes: l'ambitieuse réflexion qui décide du statut de l'iconique dans la culture débute par une interdiction, par une intervention. Moïse, qui annonce le dieu invisible du désert, triomphe de son frère Aaron, lequel, tout d'abord sans doute un adorateur des images, n'a, à tout le moins pas empêché la fabrication du Veau d'Or. C'est aussi une théorie iconoclaste qui pénètre ainsi pour la première fois dans le monde et dont les ondes de choc peuvent s'observer jusqu'à nos jours dans les tempêtes, batailles et autres effondrements des images.

2. Platon transforme en un discours de marginalisation le respect sous-jacent pour le pouvoir des images, qui est au fondement de l'interdit de la figuration et continue de s'y montrer. Les images, avec leurs corps matérialisés et périssables, lui semblent sensibles et trompeuses, et doublement éloignées de la vérité. Avec des images, on ne saurait créer un État, pas non plus une science. Le seul garant de l'identité est le logos des Idées, que Platon déploie au-dessus de nos têtes comme un ciel d'étoiles fixes. Quoi 
que l'image puisse réaliser ou ne pas réaliser, ou tout au plus exprimer, ce ne saurait être tiré de manière fiable que d'un certain usage du langage. Ce platonisme modèle, qui va parfois jusqu'à encourager les images et qui a encore conduit récemment à quelques "philosophies de l'image", ce platonisme remplace sans sourciller l'image par la puissance contraignante du mot.

16 3. Les Pères de l'Église (par exemple Grégoire de Nysse) ont adapté le concept de logos vétérotestamentaire aux conditions du Nouveau Testament, et l'ont reformulé selon l'esprit de la Trinité. L'invisibilité et l'irreprésentabilité de Dieu le Père gagne alors par l'image du Christ, une représentation visible. Seul le Christ, représenté par l'image de son origine divine, ouvre la voie de la vérité et de la vie. Sous cet angle, l'image acquiert une justification jusque-là inconnue, parce qu'il devient possible de la fonder sur le modèle de l'incarnation christique. S'ensuit une puissante universalisation de l'iconique. D'ailleurs, le christianisme était et reste la seule religion universelle qui a étendu son pouvoir grâce à l'emblème de l'image. Pourtant, même en relation avec les images du Christ, le premier et le dernier renseignement restait le logos; le croyant n'étant, en définitive, pas celui qui observe l'icône, mais celui qui reçoit la parole.

À partir de cette source et d'autres s'est développée une compréhension séculière de l'image. Depuis le XIX ${ }^{e}$ siècle, elle s'accompagne d'une utilisation massive, voire d'un véritable désir d'images, sans que cela n'ait entraîné un détournement du primat du langage, du mot ou du texte. Même dans l'usage quotidien que nous faisons des images d'information, des images fugitives des caméras de surveillance, mais aussi des images d'art, on peut encore détecter certains modèles d'iconographie. Nous observons que l'attention de l'observateur est guidée, dans l'image, par des indicateurs qui renvoient à des sub- ou à des pré-textes, de quelque type qu'ils puissent être.

L'interprète habile sait percer le secret de l'image; il déchiffre ce qui s'est caché derrière le caractère manifeste du visuel. Il démasque l'iconique comme déformation cryptée de quelque chose qui avait d'abord été dit. Qui pourrait nier que la tentative de soumettre l'image à des textes tous différents appelle souvent une nouvelle manière de voir. Il n'en reste pas moins que, dans de telles interprétations, la logique de l'image tient seulement la place d'une logique tout autre : celle de la graphé, qui se structure en une écriture de l'image (icono-graphie) dans laquelle l'iconique est guidé le long des ficelles d'un texte invisible. Ceci est toujours méthodiquement recommandé et légitime lorsqu'il s'agit de lire des images comme des substituts, c'est-à-dire comme des documents d'un sens qui s'y reflète, sans pour autant avoir été fondé par elles. Dans le cadre d'un tel regard sur les images, on aimerait manifestement savoir quelque chose. On aimerait s'approprier l'image comme porteur d'un "message ", qui est transmis d'un lieu donné vers un autre. C'est ainsi que l'historien italien Carlo Ginsburg a décrit l'image comme refuge d'un texte caché, et l'observateur comme un Sherlock Holmes. Même les images vides des caméras de surveillance ne deviennent d'ailleurs intéressantes que lorsqu'il s'y passe quelque chose, lorsque quelque chose arrive et que l'iconique retrouve son histoire par laquelle elle se justifie avant tout.

19 Il n'est nullement étranger aux images de rendre des prestations de service. Elles le font au contraire avec une telle perfection que certains auteurs et le célèbre homme de la rue ne veulent même pas envisager qu'elles aient encore un autre dessein que celui de l'illustration. Mais celui qui souligne par trop fort le texte derrière l'image atterrit invariablement dans la domination du langage, qui méconnaît les possibilités de l'image. La pratique de l'image la plus répandue et, historiquement, celle qui a le plus 
de succès, est en même temps la plus faible : elle sollicite l'image en tant que copie [Abbild]. Elle compte sur des choses ou des états de faits qui se reflèteraient en outre dans des images. Une opinion (erronée) qui est toujours répandue est également celle que la photographie ou le film seraient des garants irréfragables de cette utile duplication du monde. C'est bien sûr une tout autre prémisse qui vaut pour les images : pas un seul objet dans le monde ne prescrit la forme sous laquelle il devrait être adéquatement représenté. La supposition que la photographie serait copie est d'ailleurs réfutée entre autres par les travaux d'artistes tels que Thomas Demand, ou par la manipulation de photographies digitales (fig. 4). Ainsi, une chose est sûre : ce n'est pas par la voie de la substitution que les possibilités originelles de l'image sauraient être saisies.

$\mathrm{Au} \mathrm{Xx}^{\mathrm{e}}$ siècle, après Kant et Nietzsche, la philosophie s'est tournée vers la critique du langage. Chez Husserl, Freud, Wittgenstein, Heidegger, Merleau-Ponty, Derrida et Castoriadis, on peut observer, entre autres, deux pas : le premier veut démontrer que toute connaissance dépend en principe du langage, afin de faire basculer les fondements de la métaphysique et de l'objectivisme. Ce tournant, dans sa forme précise donnée par la philosophie analytique, a été décrit comme linguistic turn. Le concept fut employé pour la première fois en 1967 par le philosophe américain Richard Rorty comme titre d'une anthologie. Le second pas consiste en ce que la vérification de la capacité du langage conduit à son dépassement. Dans cette perspective, le linguistic turn verse en toute logique dans l'iconic turn, car dès qu'il s'agit de fonder la vérité de propositions, il faut avoir recours à des moyens extra linguistiques. L'action d'indiquer ou de montrer est redécouverte comme base de la parole. Le potentiel imagé du langage revient à une métaphorologie, telle que l'a, par exemple, développée le philosophe Hans Blumenberg, s'appuyant entre autres sur Nietzsche, comme en étant la racine authentique. Pour eux, les concepts sont des métaphores refroidies.

21 La redécouverte de l'iconique dans la fondation de la connaissance philosophique et scientifique est un processus remarquable pour la théorie de l'image. Parmi ce vaste champ, j'aimerais, dans ce qui suit, solliciter seulement deux auteurs, avec à chaque fois une réflexion essentielle, et préparer du même coup ma prochaine et ultime étape argumentative.

Le prae-nominale sur lequel se basent les propositions a été identifié par Husserl dans le «monde de la vie » [«Lebenswelt»], par Freud dans le flux de représentations qui fait apparaître la pensée et le jugement comme des "processus secondaires ", ou encore par Wittgenstein dans le concept de l'« analogie familiale ». Cela revient à dire que les propositions langagières, le logos donc, ne se laissent pas consolider en elles-mêmes. C'est tout particulièrement Wittgenstein qui a montré que la plausibilité des concepts langagiers repose sur le caractère intuitif et imagé de la langue du quotidien. C'est ainsi qu'il s'est par exemple interrogé, dans les Recherches philosophiques, sur le concept de « jeu » qui apparaît en tant que jeu de dames, de cartes, de balle, de combat, de football, de handball etc. Qu'est-ce qui fonde cette identité, qu'est-ce qui est commun à tous de telle sorte que l'on puisse parler au singulier d'« un » jeu?

«[...] si tu les regardes, tu ne verras certes pas quelque chose qui serait commun à tous, mais tu verras des similitudes, des parentés, et même toute une série. Alors, encore une fois : ne pense pas, mais regarde ! $»^{3}$ concepts pour entreprendre la fondation des savoirs; il ramène la proposition à des 
contextes flous ou, plus précisément-pour le dire dans les termes de son contemporain autrichien Robert Musil -, le sens du réel au sens du possible ; les deux n'étant que des aspects différents du même scepticisme langagier. Le « lit du fleuve des pensées » ["Flussbett der Gedanken »] n'est pas lui-même de nature langagière; la critique du langage restitue aux images qui se trouvent en nous et dans le langage quotidien leur droit fondateur et leur fonction indicatrice. Ce sont des évidences intuitives et iconiques qui aident la langue à réaliser ses possibilités. Ici, apparait un déplacement qui fait époque : le logos cesse de dominer la potentialité de l'image pour concéder sa dépendance par rapport à elle. L'image trouve accès au cercle intérieur de la théorie, auquel incombe la fondation de la connaissance.

Edmund Husserl est parvenu à ce même résultat à sa manière, avec sa phénoménologie. Son principal travail philosophique fut l'examen méticuleux de l'Anschauung et de l'intentionnalité. Il s'est, entre autres, posé la question de savoir pourquoi nous parlons sans cesse de choses (par exemple d'une armoire), alors que nous n'en voyons toujours que le devant. L'envers du monde est pour nous constamment invisible. Et pourtant, nous avons raison de penser que nous ne vivons pas pour autant dans des coulisses derrière lesquelles il n'y a rien. C'est dans ce contexte que Husserl a découvert l'horizon; non pas celui dans le lointain, mais un horizon très proche. Il a montré que toute chose "se nuance" [schattet sich $a b$ ], ce qui veut dire qu'elle s'intègre dans un contexte, de telle sorte que nous pouvons la saisir comme la perception d'un donné, d'une chose. C'est grâce à cet horizon invisible que nous pouvons présentifier dans une vue donnée toutes les autres possibles. C'est la raison pour laquelle nous voyons des "choses", et non pas des attrapes visuelles. Je voudrais retenir deux aspects qui nourrissent le concept d'une logique des images. Le premier stipule que, dans toute perception, les choses effleurent un horizon. Mais les deux appartiennent à des classifications entièrement différentes : la chose est unique et donnée, l'horizon est fluctuant, ininterrompu et potentiel - il est l'espace du possible de ce qui apparait concrètement en lui. Le second aspect réside dans le fait que nous nous mouvons maintenant dans une argumentation dans laquelle l'indéterminé et le potentiel sont la base du déterminé. Une transformation étonnante par rapport à ce que fut le logos langagier avec son emphase.

La critique philosophique du langage a, avant tout, mis un terme à un présupposé dévastateur pour toute théorie de l'image, qui avait cours depuis Parménide jusqu'au positivisme du $\mathrm{xx}^{\mathrm{e}}$ siècle. Il énonçait ceci : ce qui ne peut se dire de manière déterminée n'a pas de réalité. Ceci recelait deux conséquences : entre la structure du langage et celle de la réalité, il existe nécessairement une égalité de valeur. L'être va aussi loin que va le langage. La logique de la prédication a, dès lors, une double valeur : elle ne connaît que le oui et le non. Elle n'a pas accès à l'indéterminé, au potentiel, à l'absent ou au néant. «Rien » n'a pas de prédicat. Or, sans le divers, le polysémique, le sensuel et le polyvalent, l'on ne saurait réfléchir véritablement sur les images.

Je choisis tout d'abord l'une des œuvres majeures du $\mathrm{xx}^{\mathrm{e}}$ siècle, La danse de Henri Matisse (1909-1910), dont la complexité lapidaire convient particulièrement à notre intention (fig. 5). On peut y observer une division de l'attention. D'une part, elle se tourne vers les figures rouges clairement formées de façon presque ornementale, et d'autre part, vers l'horizon qui les accompagne. Quelle est donc la « chose » de l'image? Ce n'est ni l'un ni l'autre, mais l'un dans l'autre. En effet, nous voyons très vite qu'une différenciation s'opère et de quelle manière, puis comment lui répond le contre- 
pouvoir d'une fusion. Nous percevons une tension énergétique dans laquelle nous voyons d'abord les figures devant le fond, avant que celui-ci - la surface bleue du ciel et la surface verte du sol - ne se déplace vers l'avant, ne prenne lui-même figure dans les champs intermédiaires vacants et n'interagisse avec les danseuses. Leur mouvement de ronde orgiastique, que l'on peut décrire, selon le thème ou la narration, comme une mise en mouvement du lieu, coopère avec un tout autre mouvement: celui d'un échange des plans de l'image. Les deux s'élèvent réciproquement, stimulés par un choix de couleurs très efficace.

Ce que nous pouvons décrire dans La danse et - cum grano salis - dans d'autres tableaux, c'est le passage d'un agencement figé, dans lequel le figuratif se trouve devant le contexte, à un processus d'interaction qui fait naître du sens. C'est l'accentuation du performatif qui met en mouvement la production de sens iconique.

Lorsque nous parlons d'images (bidimensionnelles, plastiques, techniques, spatiales), nous désignons une différence dans laquelle un ou plusieurs accents thématiques [Fokus], qui attirent notre attention, se rapportent à un champ non thématique. Nous voyons l'un dans l'autre. Cette forme de relation peut, le cas échéant, être à double sens, en dépit du fait que nous soyons plutôt habitués à lire les images comme des sens uniques optiques : du proche vers le lointain, des choses vers leurs arrière-plans vides. De fait, ce qui se présente à notre regard n'est qu'une vue [Ansicht] qui est organisée en direction de l'œil et qui, pour ainsi dire nous regarde en retour.

Pourtant, ce qui est décisif pour la logique de l'image n'est pas simplement ce constat visuel, mais ses implications catégorielles. Elles stipulent que toute image tire sa force de détermination de sa liaison avec l'indéterminé. Nous ne pouvons faire autrement que d'observer le représenté par rapport à son horizon ou son contexte pré-structuré en lui. Mais celui-ci appartient à une classe catégorielle fondamentalement autre. C'est par conséquent la contamination visuelle de ces deux réalités différentes qui donne l'impulsion au fait qu'un état de fait matériel apparaisse comme image, et que naisse ce surplus de l'imaginaire dont nous avons déjà parlé en introduction. La "différence iconique " présentifie une règle de la discrimination, du contraste visuel, qui recèle simultanément un voir-ensemble. Des synthèses iconiques sont déjà disposées dans la structure de notre perception. Nous n'avons rien fait d'autre que d'adapter à l'iconique le constat de la focalisation optique dans un champ de vision mouvant - il n'y a pas d'autre manière de voir pour l'homme. Certes, les images sont toujours aussi des fixations, elles laissent se pétrifier la vie en matière, et la ressuscitent en apparence par des moyens artistiques. Mais ce qui est décisif pour que naisse le sens c'est de raviver dans l'image l'acte de voir, qui y est latent. Seule l'image vue est en vérité devenue entièrement image.

30 Il nous restera à vérifier si la thèse de la différence iconique peut être généralisée. Existe-t-il des images sans champ visuel, ou sans une thématisation focalisante? Qu'en est-il par exemple des structures « all over » (fig. 6) ? Celles-ci visent bien à niveler la composition fond-figure au profit d'une densité d'apparition. Le "tout sans parties" que ces images se voulaient de fonder ne nous atteint pas comme un éclair, mais veut lui-même être expérimenté, appréhendé à travers un acte. Dans ses peintures de camouflage, Andy Warhol a ironisé sur le pathos de la totalité de Pollock et de l'art américain de l'immédiat après-guerre.

31 La peinture de camouflage militaire sert à la soustraction visuelle. Sans différenciation, elle laisse disparaître quelque chose au profit d'un all over qui cache. L'iconique s'y 
dissout dans un continuum des choses apparemment recréé. De telles images de camouflage servent en quelque sorte à aveugler le spectateur, à l'empêcher de voir, en cherchant justement à réduire autant que possible la différence iconique.

Les images scientifiques, dont il a déjà été question en introduction, sont un tout autre champ d'épreuve. Elles s'intègrent parfaitement dans le débat, dès lors que l'on a compris que le champ visuel est toujours déjà soumis à une structuration spatiale, c'està-dire à des chiffres. Le logos iconique est étroitement apparenté au logos du chiffre (lequel, pour sa part, ne recourt pas au langage pour se fonder). Sans doute est-ce là une découverte qu'avaient déjà faite les artistes anonymes de la préhistoire, lorsqu'ils s'efforçaient de représenter des proportions et des relations complexes. Dans cette optique, l'image quadrillée, qui a été développée au cours de la Renaissance dans un contexte de rationalisation de l'espace et de contrôle des techniques de présentation et de représentation, en est déjà une conséquence très tardive. Elle représente une station sur un long chemin, sur lequel apparaîtront bien plus tard la géométrisation abstraite et la gamme rythmique comme, par exemple, dans les images de Mondrian. C'est dans une tout autre ligne de développement qu'est né, sans doute durant la seconde moitié du xvIII ${ }^{e}$ siècle, le genre de l'image cognitive qui connut le plus grand succès et qui reste omniprésente jusqu'à nos jours: le diagramme. Les diagrammes, bien qu'à forte connotation cognitive, sont de véritables images, parce qu'elles sont capables de mettre au jour une visualisation tout à fait incroyable de grandeurs chiffrées abstraites. Elles mettent en relation ce qu'il y a de plus abstrait - par exemples des données sur des volumes d'échange, de tonnages, de biens, de fréquences etc. - avec des laps temporels, et ce dans une configuration visuelle qui montre ce que l'on ne pourrait jamais lire sur de simples colonnes de chiffres. Avec le diagramme, la présentation de statistiques se trouve fondamentalement transformée. Le quantum statistique s'inverse en un quale clair et évident. Le présupposé était que le champ de l'image ne fût plus seulement considéré comme une surface structurée, mais comme fonction des coordonnées $x$ et $y$, c'est-à-dire de l'abscisse et de l'ordonnée, dont la relation mathématique est figurée sur la surface comme « solution».

D'une façon ou d'une autre, la logique des images se base sur un excédent : le factuel, en tant que ce qu'il est, se laisse voir autrement. La monstration de l'image, qui propose chaque fois à la vue un aspect, vise ce semblant visuel que nous décrivons par les termes d'effet, de plausibilité, de cognition ou d'évidence. En termes d'histoire de la culture, le passage du fait à l'agent est ce pas par lequel est née, et renaît toujours à nouveau, l'institution image, cette «scène primitive iconique » dont nous parlions au début. De la matière naît du sens, parce que les valeurs visuelles réagissent les unes aux autres à travers l'acte d'observation. Cela est mis en mouvement par une asymétrie caractéristique entre la figuration et l'horizon indéterminé, dans laquelle s'édifient également toutes les autres qualités spécifiques à l'image: vie, temporalité, affect, espace, narration, etc. Bien que ce processus puisse tout à fait être décrit, il ne devient adéquatement accessible qu'à travers l'acte de l'observation. Il s'agit d'un sens non prédicatif, qui n'est précédé par nul logos langagier, et sur lequel viennent bien sûr se greffer tous les discours langagiers requis : iconologies ou interprétations. Les images ne représentent pas un royaume clos. Mais leur culture vit du fait qu'elles affirment, face au marmonnement incessant des discours et au bruit des débats, l'étrangeté qui les habite, leur dense mutisme et leur évidente plénitude. Par-delà le langage, il existe de puissants espaces de sens, d'insoupçonnés espaces de visualité, de tonalité, de gestes, de mimique et de mouvement. Ils n'ont nul besoin d'amélioration ou de justification 
après-coup par la parole. C'est que le logos n'est justement pas que la prédication, la verbalisation et le langage. Son environnement est significativement plus vaste. Il importe de le cultiver.

\section{ANNEXES}

\section{La logique des images}

Ces mots-clés nous fournissent d'importantes indications sur le mode de fonctionnement de l'iconique. Nous allons entreprendre d'en suivre la trace, à l'aide d'exemples choisis dans le contexte de la modernité, dans le champ expérimental de laquelle presque toutes les frontières et les déterminants des images auront été déplacés et mis à l'épreuve. Que l'on songe à l'irrésistible dissolution du tableau en constellations ou en installations ouvertes, à l'énergie de l'informel faisant exploser les frontières, au passage de la sculpture vers l'objet, de l'image vers l'image en mouvement, aux photos, aux photogrammes, aux installations vidéo, etc. Si auparavant la question de l'image n'a pas été posée avec trop d'insistance, parce que la réponse semblait claire ou trop pauvre, elle apparait maintenant aussi urgente qu'infiniment plus difficile, ne serait-ce que par son changement de statut.

\section{NOTES}

1. L'ouvrage de Platon intitulé le Cratyle restitue le dialogue entre Socrate et les sophistes Cratyle et Hermogène.

2. En français dans le texte.

3. Ludwig WiTTGENSTEIn, Philosophische Untersuchungen, Werkausgabe, t. 1, Francfort/Main, 1984, paragraphe 66, p. 277 ; notre traduction.

INDEX

Mots-clés : image, langage, logique, perception

Schlüsselwörter : Bild, Logik, Sprache, Wahrnehmung 
AUTEURS

GOTTFRIED BOEHM

Professeur d'histoire de l'art moderne, Université de Bâle 